ARTICLE

\title{
Disposable silicon-based all-in-one micro-qPCR for rapid on-site detection of pathogens
}

\author{
Estefania Nunez-Bajo ${ }^{1}$, Alexander Silva Pinto Collins ${ }^{1,4}$, Michael Kasimatis ${ }^{1,4}$, Yasin Cotur ${ }^{1,4}$, Tarek Asfour ${ }^{1,4}$,
} Ugur Tanriverdi ${ }^{1}$, Max Grell', Matti Kaisti ${ }^{1}{ }^{1}$, Guglielmo Senesi ${ }^{1}$, Karen Stevenson ${ }^{3}$ \& Firat Güder (i) ${ }^{1 凶}$

Rapid screening and low-cost diagnosis play a crucial role in choosing the correct course of intervention when dealing with highly infectious pathogens. This is especially important if the disease-causing agent has no effective treatment, such as the novel coronavirus SARS-CoV2 , and shows no or similar symptoms to other common infections. Here, we report a disposable silicon-based integrated Point-of-Need transducer (TriSilix) for real-time quantitative detection of pathogen-specific sequences of nucleic acids. TriSilix can be produced at waferscale in a standard laboratory (37 chips of $10 \times 10 \times 0.65 \mathrm{~mm}$ in size can be produced in $7 \mathrm{~h}$, costing 0.35 USD per device). We are able to quantitatively detect a 563 bp fragment of genomic DNA of Mycobacterium avium subspecies paratuberculosis through real-time PCR with a limit-of-detection of $20 \mathrm{fg}$, equivalent to a single bacterium, at the $35^{\text {th }}$ cycle. Using TriSilix, we also detect the cDNA from SARS-CoV-2 (1 pg) with high specificity against SARS-

CoV (2003).

\footnotetext{
${ }^{1}$ Department of Bioengineering, Imperial College London, London SW7 2AZ, UK. ${ }^{2}$ Department of Future Technologies, University of Turku, 20500 Turku, Finland. ${ }^{3}$ Moredun Research Institute, Pentlands Science Park, Bush Loan, Edinburgh, Scotland EH26 OPZ, UK. ${ }^{4}$ These authors contributed equally: Alexander Silva Pinto Collins, Michael Kasimatis, Yasin Cotur, Tarek Asfour. ${ }^{凶}$ email: guder@imperial.ac.uk
} 
$\mathrm{D}$ espite the advancement of diagnostic technologies targeting nucleic acids (NA), there are still no rapid, handheld, low-cost, easy-to-use, and integrated solutions (i.e., amplification and detection) for the testing of infectious diseases at the point-of-need (PoN). This is unfortunately the case for pathogens infecting humans, animals, or even plants. This large gap in the diagnostic workflow hampers attempts to contain infectious pathogens from spreading by rapid and early detection. This technological gap has become, once again, evident with the spread of COVID-19, the diagnosis of which continues to depend heavily on centralized laboratories with specialized personnel and facilities, which in turn slows down testing and delays treatment.

NA make excellent targets for the direct detection of pathogens due to their high specificity. Unlike other biomarkers, such as antibodies or non-genetic molecules originating from pathogens, NA can be chemically amplified, enabling direct detection of low numbers of pathogens (down to single organisms). Hence tests targeting NA tend to be exceptionally sensitive. NA-based reagents (e.g., primers) for NA testing can also be produced synthetically rapidly and on a large scale ${ }^{1}$; DNA-based molecules, in particular, are highly stable, therefore, do not require a coldchain for storage, making it especially suitable for PoN testing ${ }^{2}$. Ability to produce NA quickly also increases the speed of development and deployment of new test kits to health systems, providing much needed diagnostic capabilities for the detection of novel pathogens (such as SARS-CoV-2). Despite the massive advantages, on-site testing of NA has been limited.

There are several molecular methods to amplify NA for detection with high specificity e.g., polymerase chain reaction (PCR), loop-mediated isothermal amplification (LAMP), stranddisplacement amplification (SDA) and recombinase-polymerase amplification (RPA). While PCR requires thermocycling, emerging isothermal methods such as LAMP and RPA do not, removing the need for sophisticated instruments ${ }^{3,4}$. On the other hand, PCR is a simple process requiring few reagents. It is also the gold standard for NA-based laboratory diagnostics with a solid support infrastructure. Amplification strategies are traditionally combined with fluorescence-based optical methods of detection to produce a quantitative analytical signal. Optical methods, even though sensitive, require expensive instruments ${ }^{5}$. The fluorescent labels used in optical detection are also difficult to handle and susceptible to photobleaching. Optical detection systems have been difficult to miniaturize and largely limited to centralized laboratories, however, some early concepts have been proposed by various groups in the literature ${ }^{6-11}$.

Despite low-cost onsite testing for infectious diseases being the holy grail of NA diagnostics, there are still no inexpensive and handheld solutions in the market that can provide truly portable, rapid NA amplification and detection. Commercially available benchtop optical lab instruments such as GeneXpert (Cepheid) have already made a substantial difference in the speed of NAbased diagnosis of infectious diseases but the high cost of the instrument/tests, large size, and power consumption have prevented the adoption of these systems for use in the field. With the sudden spread of SARS-CoV-2, multiple companies have also been developing (none commercially available yet) nucleic acid detection systems for PoN use such as Bosch Vivalytic, Abbott ID NOW COVID-19, or iAMP COVID-1912-14. These systems, however, are bench-top systems and use fluorescencebased optical analysis for detection. LamPORE by Oxford Nanopore Technologies ${ }^{15}$ aims to combine isothermal nucleic acid amplification with nanopore sequencing for the detection of SARS-CoV-2 but there is still limited data (price, performance, etc.) available concerning this technology although it is expected to be substantially more expensive than PCR-based optical approaches.
The ideal, miniaturized, low-cost portable detection system for NA must be able to heat up the sample from the patient to the desired temperature setpoint with high precision (using a heater and temperature sensor) and measure the results of the amplification reactions quantitatively, all in an integrated fashion. Using cleanroom-based semiconductor fabrication methods, such systems have been reported ${ }^{16-20}$; however, these devices require advanced methods of microfabrication that can only be performed in a cleanroom, such as photolithography, vacuum etching/deposition, etc. They also do not exploit the intrinsic properties of the semiconductor materials that are used as substrates (i.e., $\mathrm{Si}$ itself can be used both as a temperature sensor and electrical heater). Hence the devices reported are complex and expensive. Because semiconductor foundries are mainly located in East Asia, manufacturing and logistics are susceptible to disruptions in the supply chain due to unexpected events (e.g., COVID19 pandemic). There is, therefore, still no viable low-cost, rapid, quantitative, integrated (i.e., capable for NA amplification and detection) handheld NA testing solution available for use in the field with the potential for market wide availability.

Here, we show a disposable, ultra-low-cost integrated siliconbased PoN tri-modal NA transducer (TriSilix) that can chemically amplify and electrochemically detect pathogen-specific sequences of NA quantitatively in real-time. Trisilix can be produced at wafer-scale in a standard laboratory and exploits the intrinsic properties of the semiconductor Si which can be used as a resistive heating device and thermistor simultaneously. The sample solution can be maintained at a single, specific temperature (needed for isothermal amplification of NA such as RPA) or cycled between different temperatures (with a precision of $\pm 1.3^{\circ} \mathrm{C}$ ) for PCR while the exact concentration of amplicons is measured quantitatively and in real-time electrochemically. The system is operated digitally, portable, and low power-capable of running up to 35 tests with a 4000 mAh battery (a typical battery capacity of a modern smartphone). We demonstrate that TriSilix can quantitatively detect $20 \mathrm{fg}$ of a $563 \mathrm{bp}$ fragment (Insertion Sequence IS900) of genomic DNA from Mycobacterium avium paratuberculosis (extracted from cultured field samples) and $1 \mathrm{pg}$ of the cDNA from SARS-CoV-2 (1 pg) with high specificity against SARS-CoV (2003).

\section{Results}

Fabrication of TriSilix chips. To create an ultra-low-cost device, the architecture proposed here exploits the intrinsic properties of silicon and integrates three modes of operation in a single chip: (i) electrical (Joule) heater, (ii) thermistor with a negative temperature coefficient that can provide the precise temperature of the sample solution during reaction, and (iii) label-free electrochemical sensor for detecting target NA with methylene blue as a redox-active reporter. (Fig. 1 and Supplementary Video 1).

To achieve cleanroom-free, low-cost fabrication, we have developed a series of fabrication methodologies based on wet etching to form porous Silicon, electroplating, thermal bonding, and laser-cutting. The fabrication of multilayered, composite TriSilix chips (dimension of each chip: $10 \times 10 \times 0.65 \mathrm{~mm}$ ) starts with a $\mathrm{Si}$ wafer ${ }^{21}$. In this study we used lightly doped p-type 4inch $\mathrm{Si}$ wafers, however, wafers with a larger diameter would also work.

First, each wafer is plated electrolessly for $20 \mathrm{~s}$ (in $80 \mu \mathrm{M}$ $\mathrm{KAuCl}_{4}$ and $0.5 \% \mathrm{HF}$ ) to form a thin layer of gold particulate film. The wafer is then placed inside an etching bath containing an aqueous solution of $\mathrm{H}_{2} \mathrm{O}_{2}$ and $\mathrm{HF}$ with a ratio of $1: 20 \mathrm{v} / \mathrm{v}(30 \%$ $\left.\mathrm{H}_{2} \mathrm{O}_{2}: 10 \% \mathrm{HF}\right)$ to perform metal-assisted chemical etching (MACE) of Si for $10 \mathrm{~min}^{22-24}$. This process forms a $500 \mathrm{~nm}$ thick nanoporous Silicon (pSi) layer on each side of the wafer (Fig. 2a). 
a

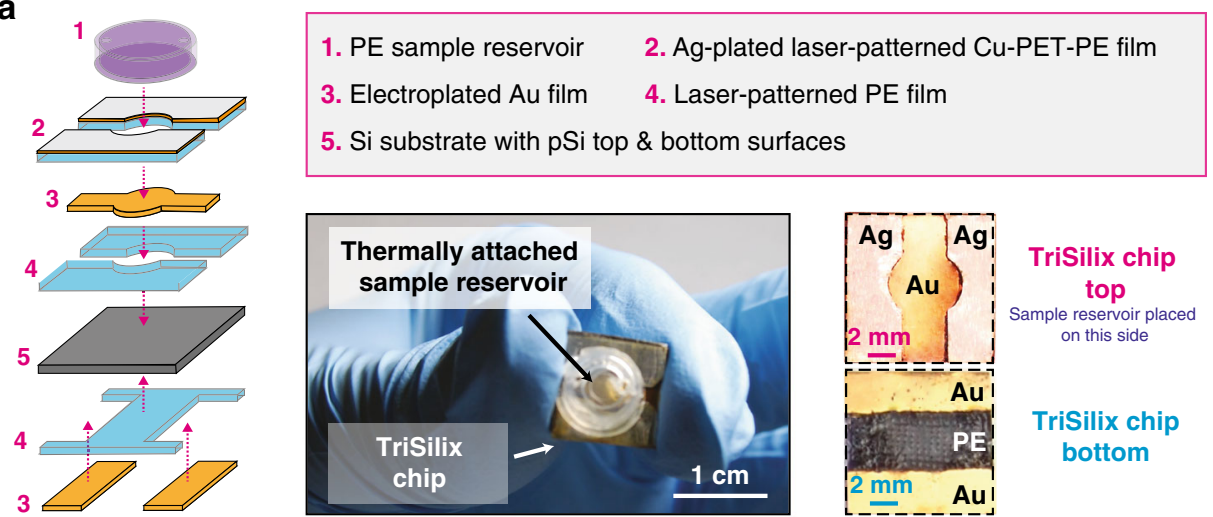

b Wafer-scale fabrication of TriSilix chips

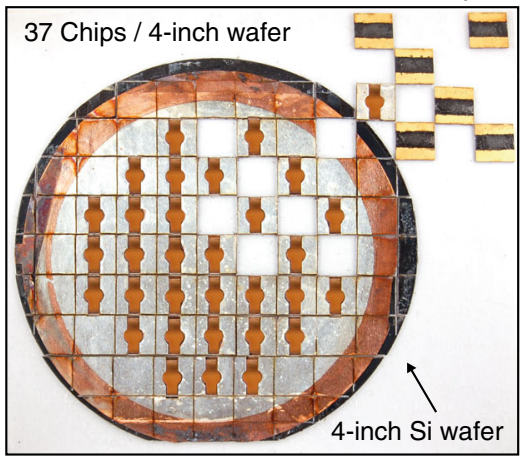

C

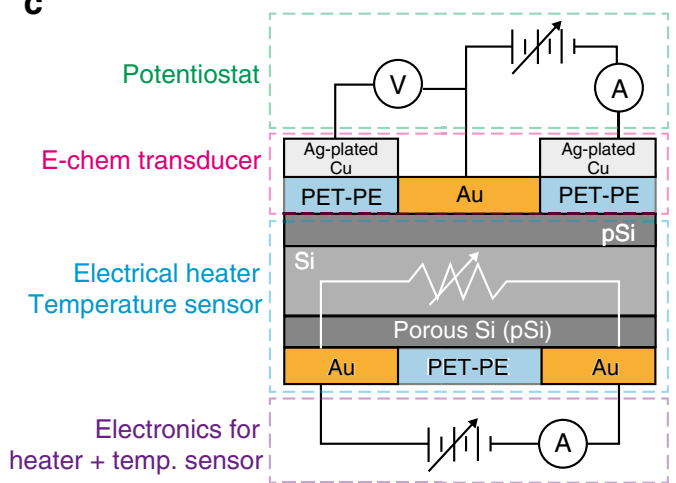

Fig. 1 Features of the proposed device (TriSilix). a Schematic illustration of construction of a TriSilix chip and photographs of the actual device. $\mathbf{b}$ Waferscale fabrication of TriSilix using a 4-inch Si wafer. Each wafer yields 37 chips. c Schematic illustration of the functional building blocks of TriSilix that provide trimodal operation for integrated nucleic acid amplification and detection.

pSi plays a critical role in the fabrication of TriSilix. The porous surface allows electroplating of high-quality metal films on the surface of the $\mathrm{Si}$ substrate by creating an interlocking, highporosity surface to improve adhesion (Fig. $2 \mathrm{~b}, \mathrm{c}$ ). Without this step, the metal films electroplated do not adhere to the surface of the substrate ${ }^{24}$. The $\mathrm{pSi}$ layer also allows thermal bonding of sheets of polymer films in an ordinary heat press after patterning through laser-cutting (Fig. 2d). After the formation of pSi surface, two layers of polyethylene terephthalate-polyethylene (PET-PE) are thermally bonded on the bottom and top surfaces of the $\mathrm{Si}$ wafer by heat pressing at $180^{\circ} \mathrm{C}$ for $5 \mathrm{~min}$. The heat pressed layers define the shape of the working electrode (WE) on the top surface and electrical contacts for Joule heating/resistance measurements on the bottom surface of the wafer. The patterned and heat pressed polymer sheets essentially act as masking layers for electroplating of the metal electrodes on the pSi surface equivalent to photolithography-based patterning in conventional microfabrication. Next, the unmasked areas of the pSi surface were cleaned in $5 \% \mathrm{HF}$ and electroplated by $\mathrm{Au}$ in a bath containing an aqueous solution of $10 \mathrm{mM} \mathrm{KAu}(\mathrm{CN})_{2} / \mathrm{KCN}$ for 10 min under a constant current of $10 \mathrm{~mA}$ versus Pt electrode $(5 \mathrm{~cm} \times 5 \mathrm{~cm})$ yielding $\sim 100 \mathrm{~nm}$ thick porous Au films. To achieve high uniformity when electroplating across the wafer, we have designed and 3D printed a custom electroplating platform that allows circular electrodes to be attached around the perimeter of the Si wafer (Supplementary Fig. 1). To form a three-electrode electrochemical cell on the top surface, the counter electrodes (CE) and reference electrodes (RE) were created by heat pressing an Ag-plated Cu-PET-PE film, patterned once again by laser cutting. Ag plating was performed in an aqueous solution of $10 \mathrm{mM} \mathrm{AgCN/KCN}$ under an applied current of $20 \mathrm{~mA}$ versus Pt electrode (using the electroplating holder). Once the basic device structure was created, the wafer was laserdiced into individual chips (37 chips / 4-inch Si wafer, Fig. 1b). We performed cyclic voltammetry $(\mathrm{CV})$ in a solution of $\mathrm{K}_{3}[\mathrm{Fe}$ $(\mathrm{CN})_{6}$ ] to study the reversibility of redox processes on the Au WE (Supplementary Fig. 2). The results indicate that the redox processes involving ferrocyanide are reversible; diffusioncontrolled processes on the WE are semi-infinite with experimental gradient values of $0.48 \pm 0.03$. Using the Randles-Sevcik equation, we also determined that the Au film has a $2 \times$ larger electroactive area than the geometrically defined area due to the high porosity of the Au film, which is beneficial for highperformance electrochemical analysis. A sample reservoir (polyethylene; diameter: $5 \mathrm{~mm}$; volume: $40 \mu \mathrm{L}$, Fig. 1a) with two holes was thermally bonded across the three-electrode electrochemical cell at $110 \pm 10^{\circ} \mathrm{C}$. The sample reservoir is specifically designed to prevent the evaporation of the solvent during amplification of DNA at elevated temperatures. The final Trisilix chip has a thickness of $490 \mu \mathrm{m}(N=7)$ in the WE region and $650 \mu \mathrm{m}$ in the $\mathrm{RE} / \mathrm{CE}$ region $(N=7)$ without the sample reservoir. Figure 1c shows a schematic illustration of the functional building blocks of TriSilix that provide trimodal operation: The three-electrode electrochemical cell fabricated on the top surface is connected to an electrochemical reader (potentiostat) to provide an electrochemical signal concerning the detection of NA (Mode 1). The electrodes fabricated at the bottom of the device are connected to custom electronics to enable heating (Mode 2) and temperature sensing (Mode 3) for thermoregulation.

Characterization of temperature transduction. NA amplification reactions require maintaining the sample at a temperature 

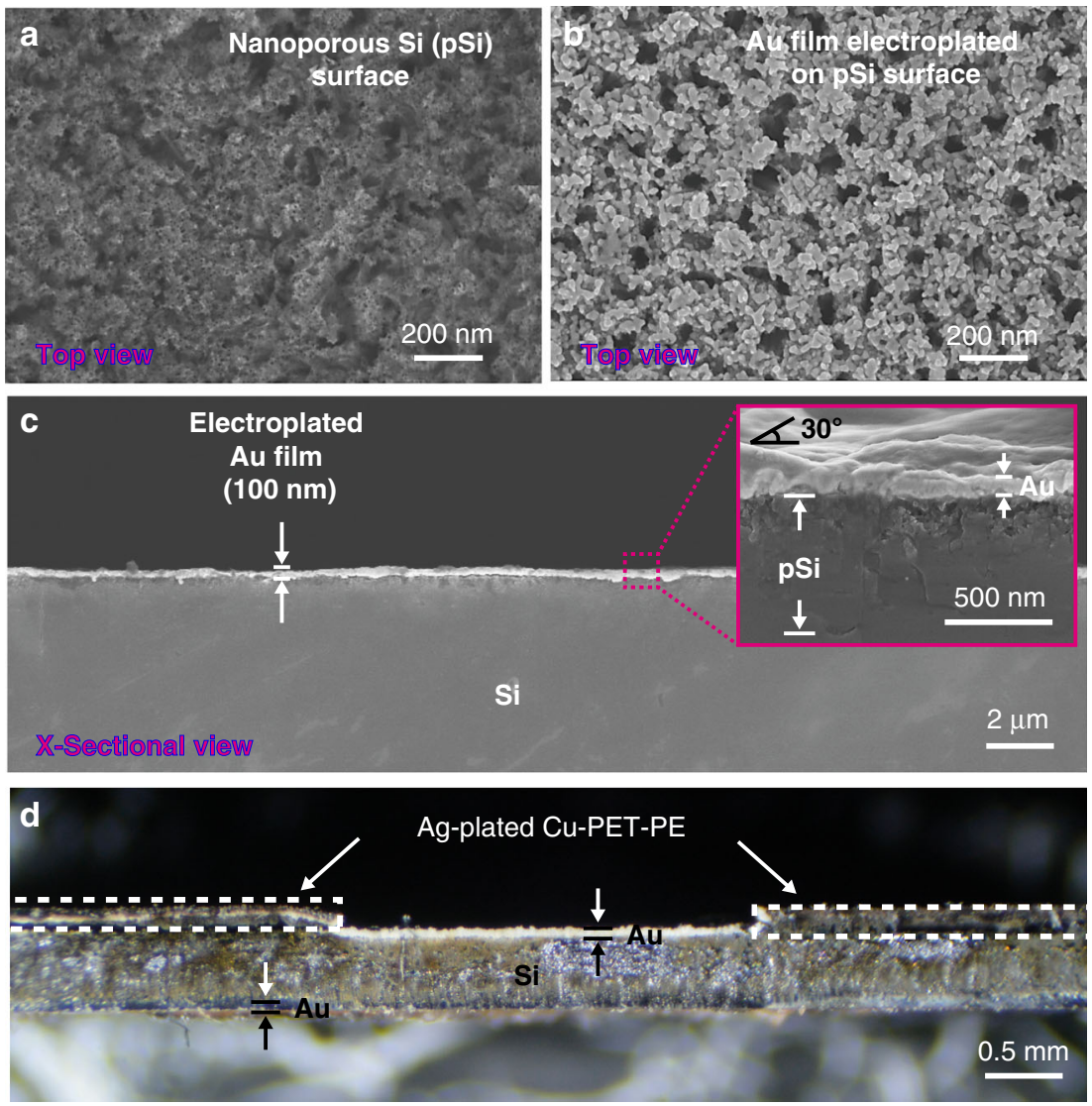

Fig. 2 Scanning electron micrographs. a Surface of Si wafer after metal-assisted chemical etching with Au catalyst producing a nanoporous Si surface (pSi). b pSi surface after electroplating with gold. c Cross-section of the sample shown in (b). The inset is a close-up view of the cross-section where the $\mathrm{Au}$ and porous Si layers are clearly visible. $\mathbf{d}$ Optical micrograph of the cross-section of a TriSilix Chip with thermally bonded sheets of polymer films. Note that while the cross-sectional image shown in (c) was cleaved, (d) was laser-cut hence the roughness.

setpoint with high precision. For PCR, the duration of each heating step must also be carefully controlled. Because TriSilix uses $\mathrm{Si}$, the substrate itself can be used both as an electrical heater and temperature sensor (i.e., thermistor) without adding any additional components for temperature transduction. $\mathrm{Si}$, a semiconductor, heats up when an electrical current passes through it (known as Joule heating). Because Si has a high thermal conductivity $\left(\sim 150 \mathrm{~W} \mathrm{~m}^{-1} \mathrm{~K}^{-1}\right.$ at $\left.300 \mathrm{~K}\right)$, the substrate can be heated uniformly regardless of the path the current flows. The electrical resistance of $\mathrm{Si}$ also varies with temperature with a negative correlation (within our experimental range of temperatures from RT to $110^{\circ} \mathrm{C}$ ); the electrical resistance of Si drops with increasing temperature due to generation of mobile charge carriers allowing the use of $\mathrm{Si}$ substrate itself as a sensitive sensor of temperature $25-27$.

We have applied electrical currents in the range $0-400 \mathrm{~mA}$ between two Au electrodes deposited on the bottom of TriSilix chip to heat up the device electrically (the experiments were performed at room temperature; $\sim 25^{\circ} \mathrm{C}$ ). During this experiment, we used a thermal camera (FLIR E4) to measure the temperature across the chip as a reference measurement. As illustrated in Fig. 3a, TriSilix chip can be heated up to $110^{\circ} \mathrm{C}$, electrically. The relationship between the current applied and substrate temperature was linear (with a positive slope of $232.3 \pm 8.7^{\circ} \mathrm{C} \mathrm{A}^{-1} ; R^{2}=$ $0.9971, N=5)$ at steady-state with high repeatability.

At room temperature, the electrical resistance of a batch of TriSilix chips was $5.4 \pm 0.6 \Omega(N=37)$ which varied linearly (with a negative slope of $-6.1 \pm 0.4 \times 10^{-3}{ }^{\circ} \mathrm{C}^{-1} ; R^{2}=0.9991, N=5$ ) with the temperature measured within the range from room temperature to $110^{\circ} \mathrm{C}$ (Fig. 3b). By measuring the electrical resistance of the Si substrate, using the two electrical contacts at the bottom of the chip, the temperature of the TriSilix chip can be precisely identified after calibration against a reference measurement (see Supplementary Table 1 for more information about the calibration and specifications of the TriSilix thermistor). Temperature sensing is important for correct and fast execution of a NA amplification program for at least two reasons: (i) Temperature can be maintained at a precise setpoint using a control algorithm such as proportional-integral-derivative control loop. (ii) When cooling (passively) or heating (actively), the speed of reaching a temperature setpoint can be maximized as both heating and cooling depend on the outside temperature and device packaging (e.g., the construction of the holder or cartridge). By only relying on the power applied, temperature control cannot be achieved with high precision.

Although we expected formation of Schottky barriers and nonlinear electrical characteristics across the Au-Si interface, the $I-V$ measurements (Fig. 3c) demonstrated that the junction exhibits relatively ohmic behavior with a linear $I-V$ relationship $\left(R^{2}=\right.$ $0.9996)$ between -5 and $+5 \mathrm{~V}$. The $\mathrm{Si}$ substrate plus the $\mathrm{Au}$ contacts can, therefore, be modeled as a simple variable resistor, the magnitude of which changes with temperature. We have designed a custom electrical circuit (Fig. 3d) and MATLAB-based graphical user interface (Supplementary Fig. 3) to control the temperature of the TriSilix chip at a precise setpoint through software control. The circuit (Fig. 3d) implements a voltage controlled constant current source which can be adjusted digitally using a low-cost microcontroller (Arduino Due). This circuit can 

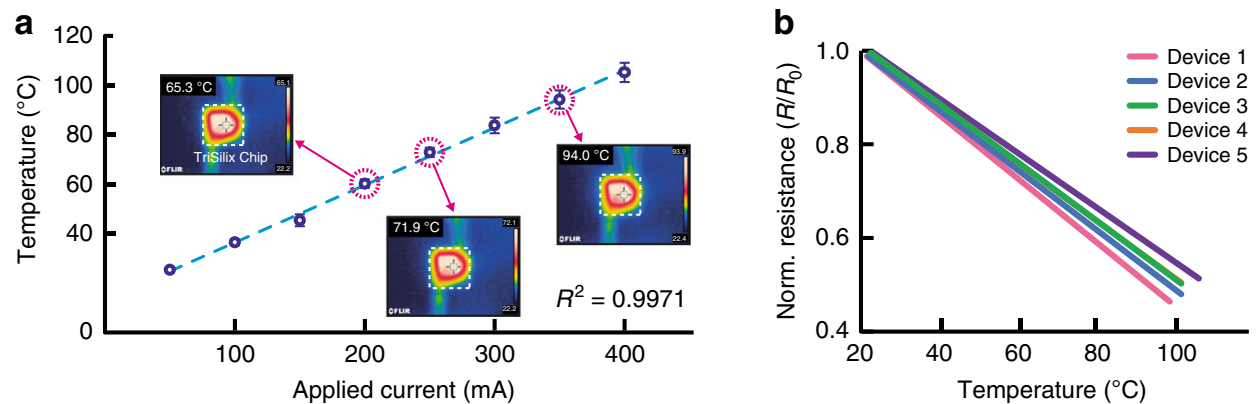

C

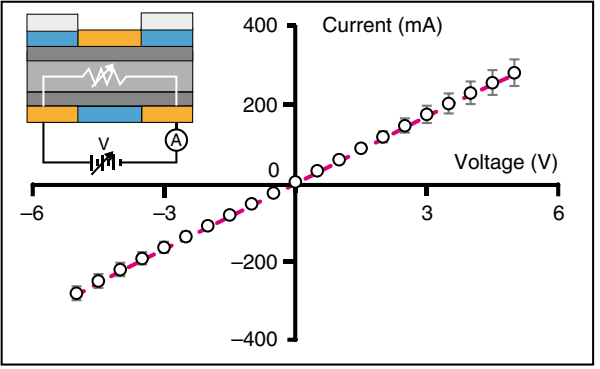

d
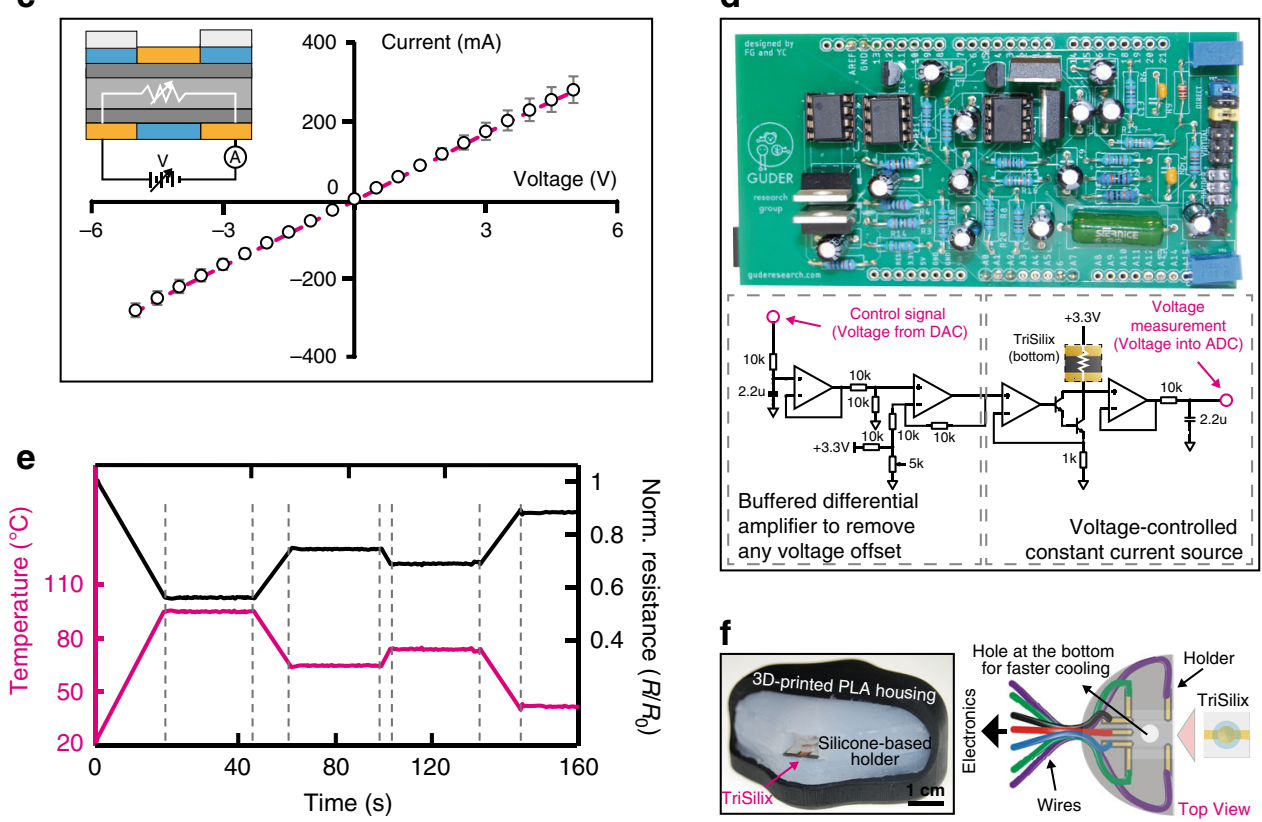

Fig. 3 Characterization of temperature transduction. a Relationship between temperature and (constant) current applied in electrothermal heating $(N=5)$. The temperature measurements were cross correlated with a thermal camera as a reference measurement. $\mathbf{b}$ Temperature vs normalized resistance (resistance/initial resistance) linear relationship showing a negative slope $(N=5)$. c $l-V$ measurements across the Au-Si-Au junction which yielded a linear, ohmic relationship between -5 and $+5 \vee\left(R^{2}=0.9996, N=5\right)$. d Photograph (top) and simplified circuit schematic diagram (bottom) of the custom electronics used for heating and temperature sensing. e Temperature program to maintain the TriSilix chip at a temperature setpoint over time using software control and custom electronics. f Photograph (left) and schematic illustration (right) of the silicone-based holder with embedded contacts to run TriSilix in trimodal operation. In all experiments shown in this figure, each repeat is performed with a new TriSilix chip and error bars correspond to standard deviations between repeats.

also measure the voltage drop across the TriSilix chip; when a constant current is applied, the resistance (hence the temperature) of the TriSilix thermistor can be calculated using the voltage reading, and Ohm's law. Using the MATLAB program and the custom electrical circuit board, we were able to run a temperature program (Fig. 3e) and maintain the TriSilix chip at a given temperature setpoint over time. This is crucial for both cyclic and isothermal NA amplification reactions.

Characterization of electrochemical sensing. We produced a thermally stable silicone-based holder encased in a 3D printed polylactic acid (PLA) housing (Fig. 3f and Supplementary Fig. 4) to characterize the performance of electrochemical sensing using the TriSilix chip. The silicone holder also contained five embedded gold-plated stainless-steel electrodes to make electrical contacts with the three electrodes positioned at the top for electrochemical NA sensing and four at the bottom for heating and temperature sensing.

First, we characterized the electrochemical redox processes for methylene blue (MB) using cyclic voltammetry (Fig. 4a). We chose MB because the electrochemical approach we used for the detection of DNA involves the use of $\mathrm{MB}^{28-30}$ as an intercalating redox reporter. During NA amplification, $\mathrm{MB}$ is intercalated between guanine-cytosine base pairs of the double-stranded DNA (ds-DNA) which provides an electroanalytical signal correlated with the concentration of ds-DNA in the sample. We prepared a $125 \mu \mathrm{g} \mathrm{mL}^{-1}$ solution of $\mathrm{MB}$ in $10 \mathrm{mM}$ phosphatebuffered saline $\mathrm{pH} 7$ (PBS) and swept across a range of potentials between -400 and $200 \mathrm{mV}$ at a scan rate of $100 \mathrm{mV} \mathrm{s}^{-1}$ to characterize the electrochemical processes involving $\mathrm{MB}$ and electrodes. We determined that the anodic peak current, originating from the oxidation of $\mathrm{MB}$ on the electrode surface, appears at $-67 \pm 2 \mathrm{mV}$ and cathodic peak (due to reduction) at $-97 \pm 4 \mathrm{mV}$ versus Ag (Fig. 4a). The absence of peaks with nearly overlapping anodic and cathodic curves in buffer alone indicates that the electrodes are electrochemically and mechanically stable and with high performance (e.g., low capacitive current). The relationship between the anodic and cathodic peak current densities $\left(\left|j_{\mathrm{pa}} / j_{\mathrm{pc}}\right|=2.63 \pm 0.09\right)$ for $\mathrm{MB}$ remains stable for at least ten successive cycles (Supplementary Fig. 5) despite MB being an irreversible redox molecule. $\mathrm{MB}$ is, therefore, suitable as a redox reporter for use with TriSilix which requires consecutive 

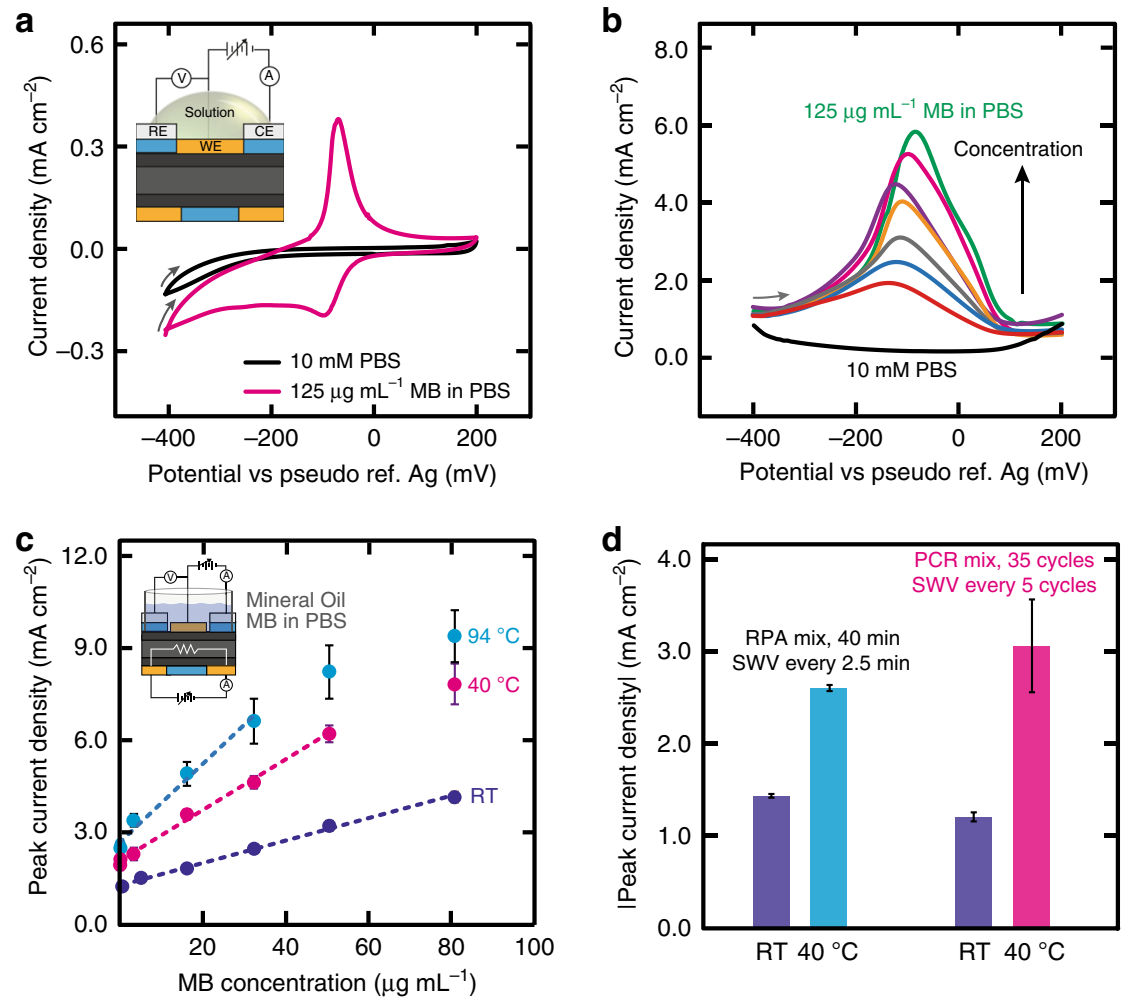

Fig. 4 Characterization of electrochemical sensing. a CVs recorded in 0 and $125 \mu \mathrm{gL}^{-1}$ aqueous solutions of $\mathrm{MB}$ in $\mathrm{PBS}$. Scan rate $=100 \mathrm{mV} \mathrm{s}^{-1}$. b SWVs recorded in MB solutions in PBS at room temperature in a range of concentrations from 0 to $125 \mu \mathrm{g} \mathrm{mL} \mathrm{m}^{-1}$. c Calibration curves of peak current densities vs concentration from SWVs recorded in MB solutions in PBS in a range of concentrations from 0 to $80 \mu \mathrm{g} \mathrm{mL} \mathrm{m}^{-1}$ at different temperatures. Error bars correspond to standard deviations from results using 5 devices $(N=5)$. $\mathbf{d}$ Histogram showing average values from SWVs recorded in RPA mastermix and PCR mastermix at room temperature and under NA amplification conditions. Error bars correspond to standard deviations from peak current intensities measured from 17 (RPA) and 8 (PCR) SWVs. In all experiments shown in this figure, each repeat is performed with a new TriSilix chip.

voltammetric measurements. We have performed (Fig. 4b) square wave voltammetry (SWV), a substantially more sensitive electroanalytical method, to measure the concentration of MB in PBS in a range between 0 and $125 \mu \mathrm{g} \mathrm{mL}-1$ using a potential window from -500 to $-250 \mathrm{mV}$ versus $\mathrm{Ag}$ (corresponding to the anodic process). The SWV measurements using TriSilix produces an electroanalytical signal (peak current intensity) that is linearly related to the concentration of $\mathrm{MB}$ in the range $0.5-80 \mu \mathrm{gL}^{-1}$ with an $R^{2}=0.9963$ ( $\mathrm{Fig}$. 4c, curve denoted by 'RT'). We have also characterized the analytical performance of TriSilix for performing SWV measurements when the chip was operated at elevated temperatures. To prevent evaporation of the solvent (i.e., water) from the sample solution, a small amount $(10 \mu \mathrm{L})$ of mineral oil was added to the reservoir. Since both isothermal and cyclic NA amplification reactions require heating, the effect of temperature on the electrochemical measurements is important. As shown in Fig. 4c, the peak current intensity (measured from the recorded SWVs) increased two to four times in comparison to room temperature when operated at higher temperatures; this is due to enhanced transport of the analyte to the surface of the electrode ${ }^{31-33}$. With increasing temperature, however, the error bars also widen, indicating that the precision of the measurement decreases. We speculate that this could be related to thermal and electrical crosstalk between the heater and the electrochemical sensing structures which introduce additional noise to the electroanalytical measurements at higher temperatures. We have also studied the thermal stability of MB over time (Fig. 4d). In this experiment, we added MB to the PCR and RPA mastermix solutions to simulate the conditions during isothermal and cyclic amplification reactions. As illustrated in Fig. 4d (and
Supplementary Fig. 6), MB remained relatively stable both at $40^{\circ} \mathrm{C}$ over 45 mins (i.e., RPA conditions) and 35 cycles of PCR conditions ( $30 \mathrm{~s}$ for each step at 94,63 , and $72^{\circ} \mathrm{C}$; every $5^{\text {th }}$ cycle, the temperature is reduced to $40^{\circ} \mathrm{C}$ for the measurement to increase precision as illustrated in Supplementary Fig. 7) indicated by small spread of the measurements. The SWV measurements at elevated temperatures produced similar results to the measurements taken at room temperature $\left(25^{\circ} \mathrm{C}\right)$ in terms of repeatability, in agreement with the findings in the literature by other groups although we did observe a slight decrease in stability for the PCR conditions (i.e., higher temperatures) ${ }^{28}$. The results of the thermal stability experiments indicate that $\mathrm{MB}$ is a sufficiently stable redox-active reporter for use in NA amplification reactions at elevated temperatures.

Real-time quantitative detection of DNA during amplification reactions. Using the TriSilix chip, we have performed real-time and quantitative RPA (isothermal, qRPA) and PCR (cyclic, qPCR) amplification of DNA using its temperature transduction capabilities and measured the concentration of reaction productssmaller fragments of ds-DNA, i.e., amplicons-using the electrochemical DNA sensor, quantitatively and in real-time. During amplification, the number of amplicons increases exponentially with time if the DNA target is present in the sample. When new amplicons are generated, $\mathrm{MB}$ interacts with the G-C pairs and can no longer participate in the electron-transfer reactions with the WE and hence the electroanalytical signal, originating from the redoxactive reporter, decreases with increasing concentration of amplicons (and the target DNA in the original sample). 
a

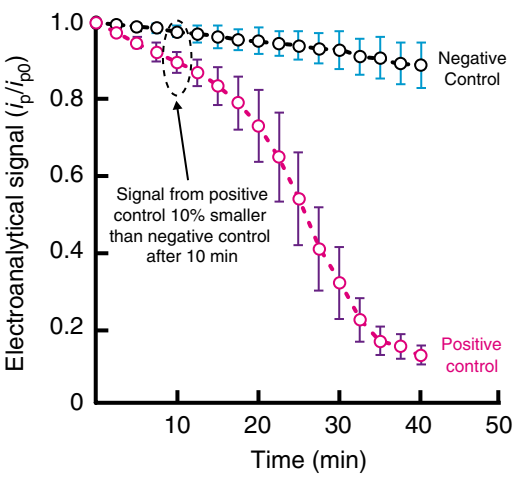

C

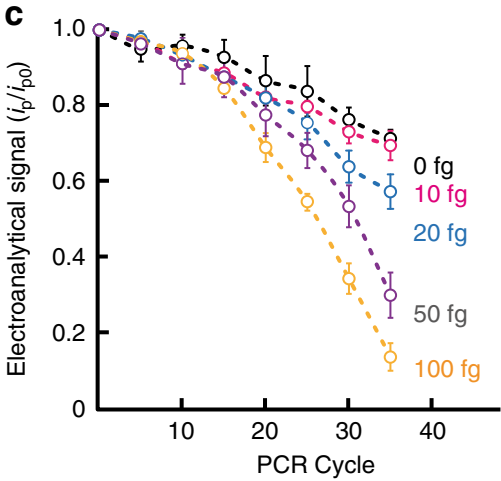

b
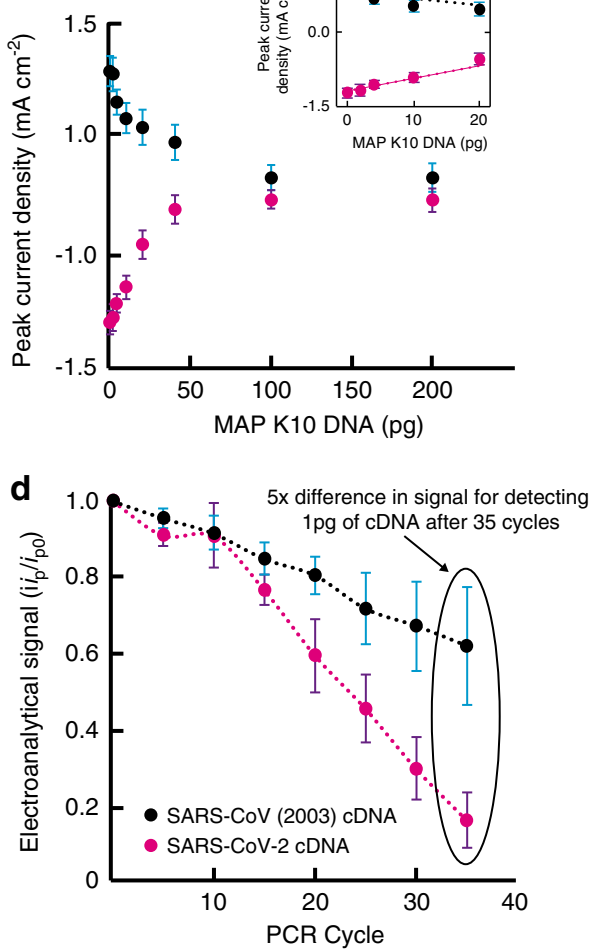

Fig. 5 Real-time quantitative NA amplification. a qRPA measurements performed using TriSilix showing a clear difference between the positive and negative control sample signals within $10 \min (N=5)$. b Titration curve for direct electrochemical detection of genomic DNA from MAP K10 DNA without amplification over 0-200 pg $(N=3)$. c qPCR measurements performed using TriSilix of genomic DNA from MAP K10 against CTMX-ESBL as negative control ( $\mathrm{Ofg}$ ). d TriSilix qPCR measurement for a synthetic fragment of cDNA from SARS-CoV-2 against a synthetic fragment of cDNA from SARS-CoV (2003) as negative sample. Clear separation between signals after 20 cycles ( 40 mins), indicating rapid, on-site detection is possible using TriSilix $(N=3)$. In all experiments shown in this figure, each repeat is performed with a new TriSilix chip and error bars correspond to standard deviations between repeats.

We performed qRPA using TriSilix with $\mathrm{MB}$ as the redoxactive reporter. We used the TwistAmp Basic kit (TwistDx, UK) with the positive (3.2 kbp template, $144 \mathrm{bp}$ amplicons) and negative (100 bp CTX-M ESBL; extended-spectrum beta-lactamases) DNA controls in the experiments. We have slightly modified the mastermix solution provided in the kit to contain $10 \mu \mathrm{g} \mathrm{mL}^{-1}$ of $\mathrm{MB}$ to enable electrochemical detection in realtime. Thirty microliter of the modified-mastermix was introduced into sample reservoir of TriSilix followed by the addition of $10 \mu \mathrm{L}$ of mineral oil to prevent evaporation. The sample was heated to $40^{\circ} \mathrm{C}$ for $40 \mathrm{~min}$ and a square-wave voltammogram was recorded every 2.5 min during the reaction. As shown in Fig. 5a, while the electroanalytical signal (normalized peak current intensity) remained relatively unchanged for the negative control, the positive control (50-150 nM according to the manufacturer) showed a large drop in signal in the form of a characteristic sigmoidal profile. This result demonstrates that TriSilix can perform real-time and quantitative DNA analysis through (isothermal) RPA reactions.

In the next experiment, we have performed qPCR analysis of genomic DNA extracted from clinical samples of Mycobacterium avium subspecies paratuberculosis (MAP) K10 strain. MAP is the causative microorganism of Johne's disease affecting ruminant animals and is controversially associated with Crohn's disease in humans. MAP is, therefore, an important analytical target which requires routine testing in centralized laboratories through microbial cultures or conventional fluorescence qPCR (lasting up to $3 \mathrm{~h})^{34-36}$. Using TriSilix, we have first performed a titration experiment to determine the limit-of-detection (LoD) of genomic DNA from MAP K10 without amplification over a range of 0-200 pg (Fig. 5b). Once again, we performed SWV as the analytical method and measured anodic and cathodic peak current intensities originating from $\mathrm{MB}$ as the electroanalytical signal. Without DNA amplification, we were able to detect genomic DNA of MAP K10 (4829 kbp) down to an LoD of $0.8 \mathrm{pg}$ from cathodic peak current intensities in comparison to an LoD of $1.2 \mathrm{pg}$ provided by the anodic peak current intensities; the cathodic peak current intensity was, therefore, used as the electroanalytical signal in the following PCR experiments. We performed qPCR using the TriSilix chip to amplify and quantify small amounts $(0-100 \mathrm{fg})$ of DNA of MAP K10 in real-time (Fig. 5c). The forward primer (5'-TGA TCT GGA CAA TGA CGG TTA CGG A-3') and reverse primer (5'-CGC GGC ACG GCT CTT GTT-3') were used to amplify a 563 nucleotide segment of IS900 (204-766 of GenBank Accession Number AE016958.1; National Center for Biotechnology Information, USA), which has 17 repeats in the genome of MAP K10. For a level of significance of $\alpha=0.05$ (2 tailed) and $d_{f}=2(N=3$, therefore degrees of freedom $\left.d_{f}=N-1=2\right)$, the application of the Student's $t$-test (see Supplementary Table 2) demonstrated that there were no statistically significant differences between the values given by qPCR for DNA solutions containing $10 \mathrm{fg}$ and blanks while there were statistically significant differences (highlighted in the table in pink) for 20,50 , and $100 \mathrm{fg}$ at the $35^{\text {th }}, 30^{\text {th }}$, and $25^{\text {th }}$ cycles, respectively. The lowest experimentally detectable concentration was $20 \mathrm{fg}$ at the $35^{\text {th }}$ cycle (Supplementary Table 2), 
equivalent to detection of a single MAP bacterium in the sample. We also characterized the same samples using a commercial laboratory qPCR (IDvet, UK) using a test manufactured by ID $\mathrm{Gene}^{\mathrm{TM}}$ as a gold-standard (Supplementary Fig. 8). The results produced by TriSilix were similar in comparison to those produced by the commercially available, sophisticated laboratory instrument; for $20 \mathrm{fg}$ of MAP K10 DNA, the resulting $C_{\mathrm{t}}$ (cycle threshold) value was 35 using TriSilix, similar to the value obtained using the commercial qPCR $\left(C_{\mathrm{t}}=35\right)$.

In a final experiment, using TriSilix, we performed $\mathrm{qPCR}$ analysis of the complimentary DNA (cDNA) of SARS-CoV-2 (22712-22869 nucleotides of GenBank accession number MN908947) ${ }^{37}$ the causative agent of COVID-19, (Fig. 5d). We have selected the forward primer $\left(5^{\prime}\right.$-CCTA CTA AAT TAA ATG ATC TCT GCT TTA CT- $3^{\prime}$ ) and reverse primer (5'-CAA GCT ATA ACG CAG CCT GTA-3') to amplify the target sequence. As a negative control, we used a cDNA fragment of another coronavirus, SARS-CoV (17741-17984 nucleotides of GenBank accession number AY274119) $)^{38}$ responsible for the SARS outbreak in 2003. Because we did not have access to patient samples, we used cDNA fragments produced synthetically by Integrated DNA Technologies Inc. We used the same PCR thermal cycling and measurement program described in the previous experiment involving MAP K10. Using TriSilix, we were able detect $1 \mathrm{pg}$ of cDNA of SARS-CoV-2 quantitatively, in realtime with specificity against a cDNA sequence from a similar virus (SARS-CoV), in as low as 20 cycles of PCR (lasting 40 $\min$ ). After 35 cycles, the difference in the electroanalytical signal was five times, showing clear separation.

\section{Discussion}

TriSilix is a low-cost, high-precision, integrated nucleic acid amplification and detection technology that is ideally suited for PoN diagnosis of infectious pathogens (bacteria, viruses, etc.) affecting, humans, animals, and plants. TriSilix can perform both isothermal and cyclic nucleic acid amplification, it integrates temperature control and DNA detection on the same device, is label-free, requires minimal sample handling, and allows operation by minimally trained personnel.

Although TriSilix is a silicon-based technology, it does not need a cleanroom for fabrication; chips can be produced in a standard wet-lab with easily accessible laboratory equipment worldwide. Because fabrication in an advanced silicon foundry is not needed, reliance on the global supply chains is substantially reduced; silicon foundries are located only in a few countries in the world. Each TriSilix chip costs $\sim 0.35$ USD (hence disposable) when produced at wafer-scale with 4 -inch wafers but the cost (cost of materials is shown in Supplementary Table 3) could be reduced further by moving to 8 -inch wafers, decreasing the size of each chip and optimizing the fabrication process (for example, the gold contacts at the bottom of the TriSilix chip could be replaced by another appropriate metal to form ohmic contacts). Due to its small size, integrated form-factor, and lowpower requirements, TriSilix can be controlled with a portable, battery-operated handheld analyzer. Using a Li-ion battery with a $4000 \mathrm{mAh}$ capacity (a typical rating for batteries in modern smartphones), TriSilix is estimated to perform at least 40 tests using isothermal amplification reactions at $40^{\circ} \mathrm{C}$ lasting $30 \mathrm{~min}$ or 13 tests using 35 cycles of PCR (these estimates assume that the electronics for the handheld analyzer also draw $50 \mathrm{~mA}$ while operating). In this work we did not optimize the cycle times for PCR (currently $90 \mathrm{~s} /$ cycle similar to standard laboratory-based PCR) but given the small thermal mass and high thermal conductivity of the TriSilix chip, there is scope for substantial reductions in cycle times.
The TriSilix technology has the following three disadvantages: (i) currently TriSilix uses $\mathrm{MB}$ as the redox-active reporter to quantify the products of the NA amplification reactions in realtime. $\mathrm{MB}$ is known to polymerize and its activity also slightly decreases over time at elevated temperatures. This issue, however, can be addressed by the use of redox-active metal-complexes that also intercalate with DNA, several of which have already been reported in the literature for real-time NA amplification ${ }^{11,28,39-41}$. (ii) TriSilix currently requires purified DNA samples for operation. (iii) Because sample reservoir and polymer films are attached thermally at $110^{\circ} \mathrm{C}$, TriSilix cannot be operated beyond this temperature without causing debonding of the layers and leaks from the sample reservoir. For most NA amplification processes, however, $110^{\circ} \mathrm{C}$ is sufficiently high. In case higher temperatures are needed (for example for on-chip sample preparation), the fabrication process would need to be modified and thermoplastics with a higher melting point could be used.

In the future, we will design a handheld analyzer and include all electronics needed for the operation of TriSilix in the same device to simplify and enable its use in the field (currently the potentiostat for electrochemical NA sensing and driver electronics for heating and temperature sensing are separate). We will also add capabilities to communicate with smartphones to have access to the cloud so that the result of each diagnostic test can be passed to health agencies remotely ${ }^{42,43}$. To enable onsite testing, we will implement on-chip lysis capabilities (such as mechanical ${ }^{44}$ or heat-induced cell lysis ${ }^{45}$ ) to directly analyze the samples taken from the subject without further sample preparation. We will also implement on-chip reverse transcription to enable analysis of RNA. TriSilix would be particularly useful in emergency situations, such as the COVID-19 pandemic, where rapid, early, on-site detection of the virus would accelerate intervention and reduce the spread of the pathogen. Since TriSilix is a low-cost, high-performance NA detection technology, it is expected to find applications beyond infectious diseases which may include genetic analysis of various plants, animals, or detection of genetic diseases ${ }^{46,47}$. TriSilix is a versatile platform and could also be adapted for electrochemical detection of non-genetic targets (e.g., through the use of antibodies) with on-chip incubation capabilities to deliver accelerated results at the PoN.

\section{Methods}

Fabrication

Preparation of $p$ Si. Silicon wafers (p-type Siegert Wafer, $525 \pm 25 \mu \mathrm{m}$ thickness, $0-100 \Omega \mathrm{cm}$, dopant density of $1.33 \times 10^{14}$ to $1.5 \times 10^{16} \mathrm{~cm}^{-3}$ ) were cleaned with acetone, rinsed with distilled water, and then immersed in a 3:1 piranha solution $\left(95 \% \mathrm{H}_{2} \mathrm{SO}_{4} / 30 \% \mathrm{H}_{2} \mathrm{O}_{2} \mathrm{v} / \mathrm{v}\right)$ at $80{ }^{\circ} \mathrm{C}$ to remove organic residues. The wafers were dried in air and immersed in a batch containing an aqueous solution of $80 \mu \mathrm{M}$ $\mathrm{KAuCl}_{4}$ and $0.5 \% \mathrm{HF}$ for $20 \mathrm{~s}$ to deposit Au particles on the surface of the wafer electrolessly. The Si wafers were rinsed with distilled water, dried, and immersed in an etching bath $\left(30 \% \mathrm{H}_{2} \mathrm{O}_{2}: 10 \% \mathrm{HF}, 1: 20 \mathrm{v} / \mathrm{v}\right.$ ratio $)$ to MACE for $10 \mathrm{~min}$. All chemicals, unless otherwise stated, were purchased from Sigma.
\end{abstract}

Patterning and heat-transfer of polymer layers. PE sheets were laser-cut (Model: Trotec Speedy 100; Wavelength: $1 \mu \mathrm{m}$; Power: $20 \mathrm{~W}$ ) and the patterns were transferred to the porous surfaces of the Si wafer (both front and back) by heatpressing at $180^{\circ} \mathrm{C}$ for $5 \mathrm{~min}$. The regions cut out were later electroplated to form the WE and electrical contacts. The Cu-PET $(20 \mu \mathrm{m} \mathrm{Cu} ; 23 \mu \mathrm{m}$ PET $)$ sheets were purchased from UK Insulations Ltd. and laminated with an additional sheet of PE film at $180^{\circ} \mathrm{C}$ for $5 \mathrm{~min}$ in a heat press to improve thermal bonding on pSi surfaces The Cu-PET-PE laminates were also laser-cut using a fiber laser, electroplated with $\mathrm{Ag}$, and heat-pressed on the top side of the Si wafer to form the electrodes for the $\mathrm{CE}$ and RE.

Electrodeposition of $\mathrm{Au}$ ang $\mathrm{Ag}$. Immediately before electroplating with Au to form the electrical contacts at the bottom of the wafer and WE on the top, each Si wafer was dipped into $5 \% \mathrm{HF}$ to remove the native oxide and produce an electrically conductive surface. The wafers were electroplated with $\mathrm{Au}$ in a bath containing $10 \mathrm{mM} \mathrm{KAu}(\mathrm{CN})_{2} / \mathrm{KCN}$ using a $5 \mathrm{~cm} \times 5 \mathrm{~cm}$ Pt electrode (SPA plating) as anode at 
a constant current of $10 \mathrm{~mA}$. The Cu-PET-PE film was first treated with $0.1 \mathrm{M}$ $\mathrm{H}_{2} \mathrm{SO}_{4}$, rinsed, and electroplated with $\mathrm{Ag}$ in an aqueous solution of $10 \mathrm{mM} \mathrm{AgCN} /$ KCN under an applied current of $20 \mathrm{~mA}$ using a $5 \mathrm{~cm} \times 5 \mathrm{~cm}$ Pt electrode as anode. All chemicals, unless otherwise stated, were purchased from Sigma.

\section{DNA amplification and extraction}

$R P A$. The RPA Premix solution was prepared by adding and vortexing $25 \mu \mathrm{L}$ of $2 \times$ buffer, $8 \mu \mathrm{L}$ of $10 \mathrm{mM}$ dNTPs (Fisher Scientific), $5 \mu \mathrm{L}$ of $10 \times \mathrm{E}$ Mix, and $5 \mu \mathrm{L}$ of control primer Mix $(30 \mathrm{bp})$ in a $0.2 \mathrm{~mL}$ Eppendorf tube. Then, $2.5 \mu \mathrm{L}$ of $20 \times$ core solution and $1 \mu \mathrm{L}$ of $0.05 \% \mathrm{MB}$ were placed in the lid of the tube and mixed with 10 inversions. The RPA Mastermix was made by mixing $3.5 \mu \mathrm{L}$ of ultrapure NucleaseFree Water (Fisher Scientific) with the Premix. This solution was subsequently mixed with $2.5 \mu \mathrm{L}$ of $280 \mathrm{mM}$ OAc and $1 \mu \mathrm{L}$ of positive control DNA or $50 \mathrm{nM}$ negative control (CTX-M ESBL: 5'-ATTGACGTGC TTTTCCGCAA TCGGATTATA GTTAACAAGG TCAGATTTTT TGATCTCAAC TCGCTGATTT AACAGATTCG GTTCGCTTTC ACTTTTCTTC-3'; Sigma) to create the final RPA mix. Then, $30 \mu \mathrm{L}$ of the final solution were added into the device chamber followed by $10 \mu \mathrm{L}$ of mineral oil (Sigma) to prevent evaporation of water. All of the chemicals (unless otherwise stated) were included in the TwistAmp Basic Kit.

MAP K10 PCRf. Fifty microliter of PCR mastermix solution in Nuclease-free ultrapure water contained $60 \mathrm{mM}$ Tris- $\mathrm{HCl}$ Buffer $\mathrm{pH} 8.8$ (Bio-Rad), $200 \mu \mathrm{M}$ dNTPs, $200 \mathrm{nmol} \mu \mathrm{L}^{-1}$ of each primer (Biomers), 0.5 U Taq DNA polymerase, and $20 \mu \mathrm{g} \mathrm{mL}^{-1} \mathrm{MB}$. Fifty microliter of PCR mix solution in Nuclease-free water contained $60 \mathrm{mM}$ Tris- $\mathrm{HCl}$ Buffer $\mathrm{pH} 8.8$ (Bio-Rad), $200 \mu \mathrm{M}$ dNTPs, $200 \mathrm{nmol} \mu \mathrm{L}^{-1}$ of each primer (Biomers), $0.5 \mathrm{U}$ Taq DNA polymerase, $2 \mathrm{mM}$ $\mathrm{MgCl}_{2}, 20 \mu \mathrm{g} \mathrm{mL}^{-1} \mathrm{MB}$, and $1 \mu \mathrm{L}$ of MAP or Nuclease-free ultrapure water. Thirty microliter of PCR mastermix or PCR mix solutions were added into the sample reservoir of TriSilix for reaction, followed by $10 \mu \mathrm{L}$ of mineral oil (Sigma) to prevent evaporation. All chemicals, unless otherwise stated, were purchased from Fisher Scientific.

SARS-CoV PCR. The PCR Mix consisted of $0.5 \mathrm{U}$ Taq polymerase, $1 \times$ Taq Polymerase buffer, $1 \mu \mathrm{M}$ of each primer (Biomers), $2 \mathrm{mM} \mathrm{MgCl}_{2}, 0.2 \mathrm{mM}$ dNTPs,

$30 \mu \mathrm{g} \mathrm{mL}^{-1} \mathrm{MB}$, and $1 \mathrm{pg} \mathrm{cDNA}$ (IDtdna). Thirty microliter of this solution were added into the sample reservoir of TriSilix for reaction, followed by $10 \mu \mathrm{L}$ of mineral oil (Sigma) to prevent evaporation. All chemicals, unless otherwise stated, were purchased from Fisher Scientific.

Extraction of genomic DNA. Genomic DNA was prepared from a $10 \mathrm{~mL}$ culture of MAP K10 in Middlebrook 7H9 supplemented with 10\% Albumin Dextrose Catalase enrichment, $0.2 \%$ (vol/vol) glycerol, $0.05 \%$ (vol/vol) Tween 80 and $2 \mu \mathrm{g} \mathrm{mL}^{-1}$ mycobactin j. Mycobacteria were harvested in early to mid log phase of growth and the cells were pelleted at room temperature for $5 \mathrm{~min}$ at $14,000 \mathrm{~g}$. Pellets were resuspended in ATL buffer (Qiagen DNeasy ${ }^{\varpi}$ Blood \& Tissue Kit) and the samples were transferred to Lysing Matrix B tubes $(0.1 \mathrm{~mm}$ silica spheres). Samples were homogenized in a FastPrep ${ }^{\text {mi }}$ FP120 cell disruptor at $3 \times 20$ s, Speed 6, followed by centrifugation at $14,000 \mathrm{~g}$ for $5 \mathrm{~min}$. Supernatants were transferred to fresh sterile micro centrifuge tubes and Proteinase K (Qiagen DNeasy ${ }^{\oplus} \mathrm{Kit}$ ) was added followed by incubation overnight at $56^{\circ} \mathrm{C}$. DNA was extracted using DNeasy ${ }^{\oplus}$ Kit (Qiagen) according to the manufacturer's protocol. Concentration $\left(40 \mu \mathrm{g} \mathrm{mL}^{-1}\right)$ and purity of the DNA were determined using the NanoDrop and the absorbance ratios 260/ 280 and $260 / 230$.

\section{Characterization}

Microstructural characterization. Scanning electron micrographs were acquired using a Zeiss Gemini Sigma 300 FEG SEM at $5 \mathrm{keV}$ electron beam energy. Optical micrographs were obtained with a Brunel SP202XM metallurgical microscope. A Nikon D3200 camera was attached to the microscope for the acquisition of the images using DigiCamControl open-source software.

Electrical and thermal characterization. The $I-V$ curves for the characterization of the metal-semiconductor junctions were acquired using a Keithley 2450 SourceMetter. Thermal images were recorded using a FLIR E4 thermal camera.

Electrochemical measurements. Cyclic (CV) and square wave (SWV) voltammograms were acquired using a handheld potentiostat (PalmSens3, PalmSens BV, The Netherlands) with the supplied PSTrace 5.3 software in a three-electrode setup. The following parameters were used for all the SWV measurements: pulse amplitude of $100 \mathrm{mV}$, potential step of $5 \mathrm{mV}$, and $50 \mathrm{~Hz}$ of frequency. Prior to the DNA measurements by SWV, the sample reservoir was cleaned with DNA decontamination reagent (Fisher Scientific) followed by Nuclease-free ultrapure water (Fisher Scientific)

\section{Data availability}

The datasets generated during and/or analyzed during the current study are available from the corresponding author on reasonable request.
Received: 9 July 2020; Accepted: 29 October 2020;

Published online: 02 December 2020

\section{References}

1. Glavan, A. C. et al. Analytical devices based on direct synthesis of DNA on paper. Anal. Chem. 88, 725-731 (2016).

2. Chen, A. \& Yang, S. Replacing antibodies with aptamers in lateral flow immunoassay. Biosens. Bioelectron. 71, 230-242 (2015).

3. Zhang, Y. \& Ozdemir, P. Microfluidic DNA amplification: a review. Anal. Chim. Acta 638, 115-125 (2009).

4. Lee, S. H. et al. Emerging ultrafast nucleic acid amplification technologies for next generation molecular diagnostics. Biosens. Bioelectron. 141, 111448 (2019)

5. Menzel, A., Gübeli, R. J., Güder, F., Weber, W. \& Zacharias, M. Detection of real-time dynamics of drug-target interactions by ultralong nanowalls. Lab Chip 13, 4173-4179 (2013).

6. Jokerst, J. C. et al. Development of a paper-based analytical device for colorimetric detection of select foodborne pathogens. Anal. Chem. 84, 2900-2907 (2012)

7. Li, Y., Cu, Y. T. H. \& Luo, D. Multiplexed detection of pathogen DNA with DNA-based fluorescence nanobarcodes. Nat. Biotechnol. 23, 885-889 (2005).

8. Donhauser, S. C., Niessner, R. \& Seidel, M. Sensitive quantification of Escherichia coli O157:H7, Salmonella enterica, and Campylobacter jejuni by combining stopped polymerase chain reaction with chemiluminescence flowthrough DNA microarray analysis. Anal. Chem. 83, 3153-3160 (2011).

9. Chen, Y. et al. In situ hybridization chain reaction amplification for universal and highly sensitive electrochemiluminescent detection of DNA. Anal. Chem. 84, 7750-7755 (2012).

10. Reboud, J. et al. Paper-based microfluidics for DNA diagnostics of malaria in low resource underserved rural communities. Proc. Natl Acad. Sci. USA 116, 4834-4842 (2019)

11. Tsaloglou, M.-N. et al. Handheld isothermal amplification and electrochemical detection of DNA in resource-limited settings. Anal. Biochem. 543, 116-121 (2018).

12. Guglielmi, G. The explosion of new coronavirus tests that could help to end the pandemic. Nature 583, 506-509 (2020).

13. Ackerman, C. M. et al. Massively multiplexed nucleic acid detection with Cas13. Nature 582, 277-282 (2020).

14. Yi-Wei, Tang et al. An Isothermal, multiplex amplification assay for detection and genotyping of human papillomaviruses in formalin-fixed, paraffinembedded tissues. J. Mol. Diagnostics 22, 419-428 (2020).

15. Peto, L. et al. Diagnosis of SARS-CoV-2 infection with LamPORE, a highthroughput platform combining loop-mediated isothermal amplification and nanopore sequencing. preprint at http://medRxiv.org 2020.09.18.20195370 (2020).

16. Fang, X. et al. Real-time monitoring of strand-displacement DNA amplification by a contactless electrochemical microsystem using interdigitated electrodes. Lab Chip 12, 3190-3196 (2012).

17. Yeung, S.-W., Lee, T. M.-H., Cai, H. \& Hsing, I.-M. A DNA biochip for onthe-spot multiplexed pathogen identification. Nucleic Acids Res. 34, e118 (2006).

18. Petralia, S. et al. A miniaturized silicon based device for nucleic acids electrochemical detection. Sens. Bio-Sens. Res. 6, 90-94 (2015).

19. Yeung, S. S. W., Lee, T. M. H. \& Hsing, I.-M. Electrochemistry-based real-time PCR on a microchip. Anal. Chem. 80, 363-368 (2008).

20. Lee, T. M.-H., Carles, M. C. \& Hsing, I.-M. Microfabricated PCRelectrochemical device for simultaneous DNA amplification and detection. Lab Chip 3, 100-105 (2003).

21. Güder, F. et al. Toward discrete multilayered composite structures: do hollow networks form in a polycrystalline infinite nanoplane by the Kirkendall effect? Chem. Mater. 23, 4445-4451 (2011).

22. Grell, M. et al. Autocatalytic metallization of fabrics using Si ink, for biosensors, batteries and energy harvesting. Adv. Funct. Mater. 29, 1804798 (2019).

23. Güder, F., Yang, Y., Kucukbayrak, U. M. \& Zahcarias, M. Tracing the migration history of metal catalysts in metal-assisted chemically etched silicon. ACS Nano 7, 1583-1590 (2013).

24. Kasimatis, M. et al. Monolithic solder-on nanoporous $\mathrm{Si}-\mathrm{Cu}$ contacts for stretchable silicone composite. ACS Appl. Mater. Interfaces 11, 47577-47586 (2019).

25. Gesele, G., Linsmeierdag, J., Drachdag, V., Frickedag, J. \& Arens-Fischer, R. Temperature-dependent thermal conductivity of porous silicon. J. Phys. D. 30, 2911-2915 (1997)

26. Weisse, J. M. et al. Thermal conductivity in porous silicon nanowire arrays. Nanoscale Res. Lett. 7, 554 (2012). 
27. Boukai, A. I. et al. Silicon nanowires as efficient thermoelectric materials. Nature 451, 168-171 (2008).

28. Deféver, T., Druet, M., Evrard, D., Marchal, D. \& Limoges, B. Real-Time electrochemical PCR with a DNA intercalating redox probe. Anal. Chem. 83, 1815-1821 (2011).

29. Fang, H. et al. Real-time PCR microfluidic devices with concurrent electrochemical detection. Biosens. Bioelectron. 24, 2131-2136 (2009).

30. Won, B. Y. et al. Investigation of the signaling mechanism and verification of the performance of an electrochemical real-time PCR system based on the interaction of methylene blue with DNA. Analyst 136 1573-1579 (2011).

31. Wildgoose, G. G., Giovanelli, D., Lawrence, N. S. \& Compton, R. G. High-temperature electrochemistry: a review. Electroanalysis 16, 421-433 (2004).

32. Watanabe, E., Mizuguchi, S., Hayashi, T. \& Toyama, K. The effect of temperature on the electrochemical properties of copper-DNA membranes. Biosensors 3, 117-125 (1987)

33. Schulzke, C. Temperature dependent electrochemistry-a versatile tool for investigations of biology related topics. Dalt. Trans. 34, 6683-6691 (2009).

34. Mobius, P., Hotzel, H., Rassbach, A. \& Kohler, H. Comparison of 13 singleround and nested PCR assays targeting IS900, ISMav2, f57 and locus 255 for detection of Mycobacterium avium subsp. paratuberculosis. Vet. Microbiol 126, 324-333 (2008).

35. Vansnick, E. et al. Newly developed primers for the detection of Mycobacterium avium subspecies paratuberculosis. Vet. Microbiol 100, 197-204 (2004).

36. Plain, K. et al. High-throughput direct fecal PCR assay for detection of Mycobacterium avium subsp. paratuberculosis in sheep and cattle. J. Clin. Microbiol. 52, 745-757 (2014).

37. Chan, J. F.-W. et al. A familial cluster of pneumonia associated with the 2019 novel coronavirus indicating person-to-person transmission: a study of a family cluster. Lancet 395, 514-523 (2020).

38. Poon, L. L. M. et al. Rapid detection of the severe acute respiratory syndrome (SARS) coronavirus by a loop-mediated isothermal amplification assay. Clin. Chem. 50, 1050-1052 (2004).

39. Deféver, T. et al. Real-time electrochemical monitoring of the polymerase chain reaction by mediated redox catalysis. J. Am. Chem. Soc. 131, 11433-11441 (2009).

40. Ferapontova, E. E. Electrochemical indicators for DNA electroanalysis. Curr. Anal. Chem. 7, 51-62 (2011).

41. Patterson, A. S., Hsieh, K., Soh, H. T. \& Plaxco, K. W. Electrochemical realtime nucleic acid amplification: towards point-of-care quantification of pathogens. Trends Biotechnol. 31, 705-712 (2013).

42. Baradun, G. et al. Cellulose fibers enable near zero-cost electrical sensing of water-soluble gases. ACS Sens. 4, 1662-1669 (2019).

43. Güder, F. et al. Paper-based electrical respiration S sensor. Angew. Chem. Int. Ed. 55, 5727-5732 (2016).

44. Hansen, S. et al. Development of rapid extraction method of Mycobacterium avium subspecies paratuberculosis DNA from bovine stool samples. Diagnostics 6, 36-44 (2019).

45. Heiniger, E. K. et al. Comparison of point-of-care-compatible lysis methods for bacteria and viruses. J. Microbiol. Methods 128, 80-87 (2016).

46. Dincer, C. et al. Disposable sensors in diagnostics, food, and environmental monitoring. Adv. Mater. 31, 1806739 (2019).

47. Bruch, R. et al. CRISPR/Cas13a-powered electrochemical microfluidic biosensor for nucleic acid amplification-free miRNA diagnostics. Adv. Mater. 31, 1905311 (2019).

\section{Acknowledgements}

F.G., K.S. and E.N.-B. would like to thank the Wellcome Trust (Grant No. 207687/Z/17/Z) and the UK Engineering and Physical Sciences Research Council (EPSRC) (EP/ R010242/1) and Imperial College, Department of Bioengineering for their generous support. K.S. also thanks Scottish Government Rural and Environment Science and Analytical Services. M. Kasimatis acknowledges EPSRC DTP (Reference: 1846144) and A.C., BBSRC DTP (Reference: 2177734). Y.C. thanks the Turkish Ministry of Education. M. Kaisti acknowledges Finnish Foundation for Technology Promotion. F.G. and M.G. thank the Imperial College Centre for Plastic Electronics. F.G. also acknowledges Agri Futures Lab. We would like to thank George Caldow from SRUC (Midlothian, UK) for performing the commercial qPCR MAP tests, and Dr. Tolga Bozkurt (Imperial College London) for the fruitful discussions.

\section{Author contributions}

E.N.-B. developed the RT-PCR-EC platform. A.S.P.C. developed the wafer holder for electroplating and performed the thermistor specifications measurements. M. Kasimatis assisted during the Si etching process and helped with SEM imaging. M. Kasimatis and M.G. helped with electrical characterization the $I-V$ curves. Y.C. and F.G. developed the custom board. Y.C. developed the software for the temperature control and sensing. U.T., T.A., and M. Kaisti developed the sample holder for TriSilix. T.A. made the animation included in supporting information. G.S. took some of the pictures presented in the manuscript. K.S. provided the genomic samples for MAP, and advice concerning the biological experiments. F.G. came up with the idea, made the figures and helped with writing.

\section{Competing interests}

The authors declare no competing interests.

\section{Additional information}

Supplementary information is available for this paper at https://doi.org/10.1038/s41467020-19911-6.

Correspondence and requests for materials should be addressed to F.G.

Peer review information Nature Communications thanks Gerald Urban and the other, anonymous reviewers for their contribution to the peer review of this work.

Reprints and permission information is available at http://www.nature.com/reprints

Publisher's note Springer Nature remains neutral with regard to jurisdictional claims in published maps and institutional affiliations.

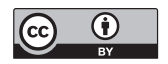

Open Access This article is licensed under a Creative Commons Attribution 4.0 International License, which permits use, sharing, adaptation, distribution and reproduction in any medium or format, as long as you give appropriate credit to the original author(s) and the source, provide a link to the Creative Commons license, and indicate if changes were made. The images or other third party material in this article are included in the article's Creative Commons license, unless indicated otherwise in a credit line to the material. If material is not included in the article's Creative Commons license and your intended use is not permitted by statutory regulation or exceeds the permitted use, you will need to obtain permission directly from the copyright holder. To view a copy of this license, visit http://creativecommons.org/ licenses/by/4.0/.

(c) The Author(s) 2020, corrected publication 2020 